\title{
Terahertz Frequency Combs Exploiting an On-Chip, Solution- Processed, Graphene-Quantum Cascade Laser Coupled-Cavity
}

Francesco P. Mezzapesa, Katia Garrasi, Johannes Schmidt, Luca Salemi, Valentino Pistore, Lianhe Li, A. Giles Davies, Edmund H. Linfield, Michael Riesch, Christian Jirauschek, Tian Carey, Felice Torrisi, Andrea C. Ferrari, and Miriam S. Vitiello*

Cite This: ACS Photonics 2020, 7, 3489-3498

Read Online

ACCESS | Llll Metrics \& More | 回 Article Recommendations | st Supporting Information

ABSTRACT: The ability to engineer quantum-cascade-lasers (QCLs) with ultrabroad gain spectra, and with a full compensation of the group velocity dispersion, at terahertz $(\mathrm{THz})$ frequencies, is key for devising monolithic and miniaturized optical frequencycomb-synthesizers (FCSs) in the far-infrared. In THz QCLs fourwave mixing, driven by intrinsic third-order susceptibility of the intersubband gain medium, self-locks the optical modes in phase, allowing stable comb operation, albeit over a restricted dynamic range ( $\sim 20 \%$ of the laser operational range). Here, we engineer miniaturized $\mathrm{THz}$ FCSs, comprising a heterogeneous $\mathrm{THz} \mathrm{QCL}$, integrated with a tightly coupled, on-chip, solution-processed, graphene saturable-absorber reflector that preserves phasecoherence between lasing modes, even when four-wave mixing

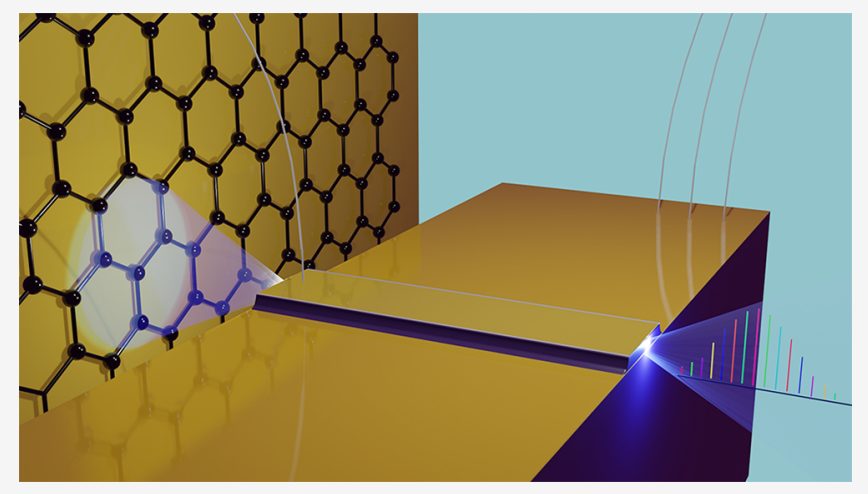
no longer provides dispersion compensation. This enables a highpower $(8 \mathrm{~mW})$ FCS with over 90 optical modes, through $55 \%$ of the laser operational range. We also achieve stable injectionlocking, paving the way to a number of key applications, including high-precision tunable broadband-spectroscopy and quantummetrology.

KEYWORDS: nanoengineered devices, integrated nanostructures, graphene, saturable absorbers, semiconductor heterostucture lasers, frequency combs

$\mathrm{O}$ ptical frequency comb synthesizers (FCSs) enable broadband coherent light sources to be developed, comprising a large number of equally spaced lasing modes. ${ }^{1}$ Chip-scale, broadband, monolithic, high brightness FCS sources at $\mathrm{THz}$ frequencies are needed for metrology, ${ }^{2-6}$ ultrahigh speed communications, ${ }^{6}$ coherent nanotomography, ${ }^{7}$ and near-field broadband nanoscopy and enable new approaches to high-resolution broadband molecular spectroscopy, manipulation of complex molecules and cold atoms, astronomy, and attosecond science. ${ }^{8}$

QCLs are the highest brightness miniaturized sources in the infrared (IR). ${ }^{9,10}$ They combine an inherently high spectral purity, ${ }^{11,12}$ with a very broad bandwidth, ${ }^{13-15}$ Watt-level output-powers, ${ }^{16}$ and a long upper-state lifetime (>5-10 ps). Broad emission in the $\mathrm{THz}$ range can be attained by quantumtailoring the gain medium to host heterogeneous stacks of individual active regions, which themselves are incorporated into a monolithic microstrip-line metal-metal resonator. ${ }^{17,18}$ The cascading design of individual emitters at complementary wavelengths creates a flat broad-gain at a desired bias point, which is usually slightly above the onset of multimode emission. The long upper state lifetime $(\geq 5-10 \mathrm{ps})^{9,10,19}$ of
THz QCLs inhibits mode-locking but favors phase-matching between the cavity modes driven by the ultrafast nonlinearity (four-wave mixing, FWM) spontaneously arising in the intersubband gain medium. ${ }^{13,15,20}$ The resulting stable frequency comb regime tends, however, to be restricted to lower injection currents, close to the onset of multimode emission, owing to the intersubband bias-dependent contribution to the group velocity dispersion. ${ }^{13,15,20}$ The heterogeneous nature of the gain media then entangles the dispersion dynamics at other biases. ${ }^{20}$ Tailoring the dynamic (biasdependent) contribution to the chromatic dispersion is, therefore, crucial for establishing perfectly spaced, phaselocked, high-intensity modes spanning the entire dynamic range of the laser.

Received: September 29, 2020

Published: December 3, 2020 

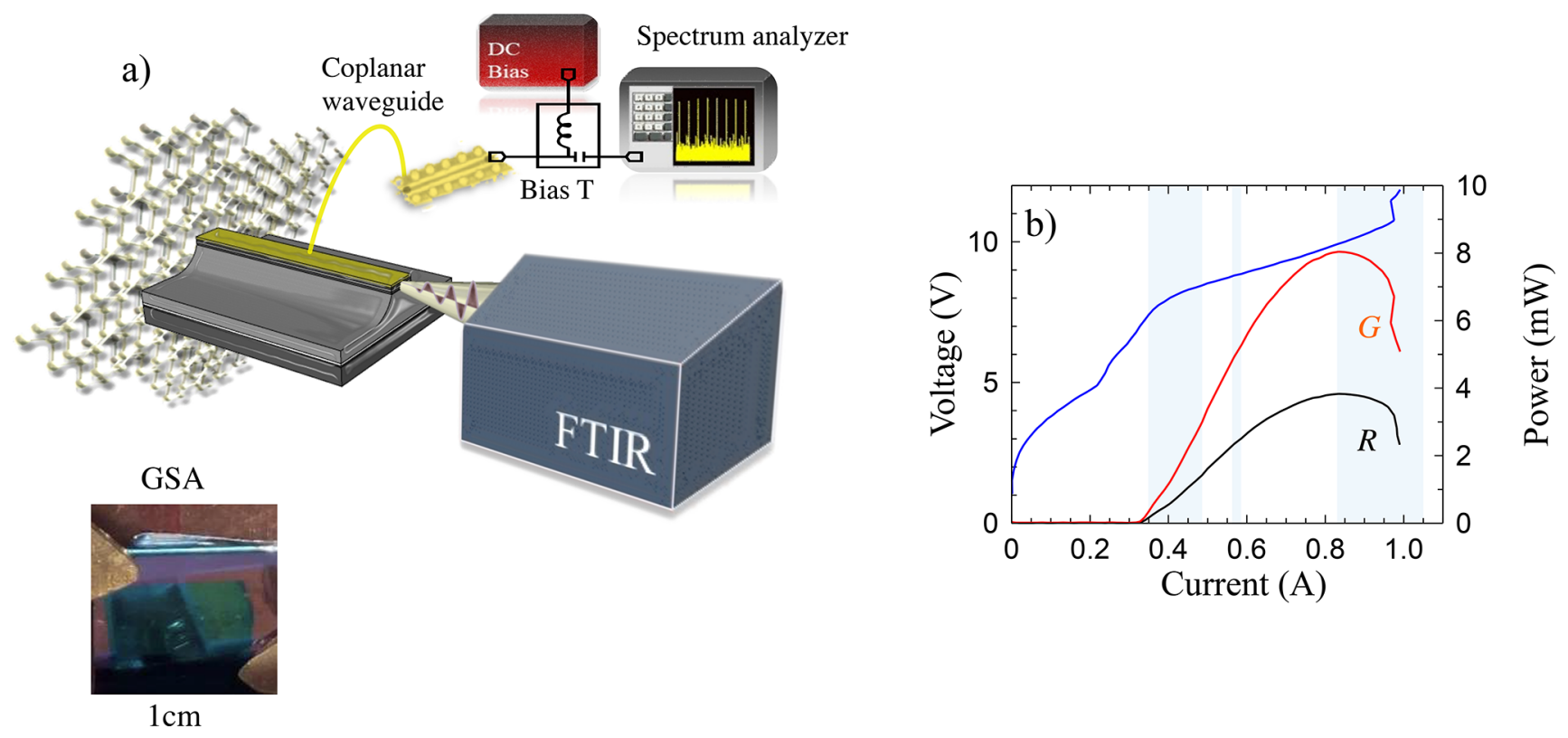

Figure 1. THz QCL with an on-chip graphene saturable absorber (GSA) reflector. (a) Schematic of a THz QCL comb coupled on chip with a GSA reflector; (b) Continuous-wave (CW) current-voltage (I-V) and light-current (L-I) characteristics of a $3.6 \mathrm{~mm}$ long, $50 \mu \mathrm{m} \mathrm{wide} \mathrm{THz} \mathrm{QCL} \mathrm{at} \mathrm{a}$ heat sink temperature of $15 \mathrm{~K}$, without $(R)$ and with $(G)$ the on-chip GSA reflector. The optical power is measured with a calibrated power-meter (Thomas Keating Ltd.) and corrected to take into account the $75 \%$ absorption of the cryostat window. The light blue shaded areas correspond to the regimes in which the laser behaves like an optical frequency comb synthesizer.

The use of chirped mirrors ${ }^{20}$ and/or coupled cavities ${ }^{21-23}$ was proposed for group velocity dispersion (GVD) compensation in homogeneous ${ }^{20,21}$ and heterogeneous ${ }^{22,23} \mathrm{THz}$ QCL FCSs, respectively. In the first case, the corrugation length and the tapering period of a chirped cavity was designed to optimize the anomalous group delay dispersion (GDD) in a specific, narrow, range of biases, leading to an FCS extending over $24 \%$ of the laser dynamic range, covering a $0.6 \mathrm{THz}$ spectral bandwidth, but with an uneven distribution of power among the 60 modes. $^{20}$ In the second case, a coupled cavity, mimicking a Gires-Tournois interferometer (GTI), ${ }^{21}$ was monolithically integrated in front of the $\mathrm{THz}$ QCL cavity, introducing chromatic dispersion that compensates for that in the gain medium. ${ }^{21}$ When DC biased, the small coupled-cavity section gave rise to an FCS over the whole QCL dynamic range, although there was only a very limited amount of optical power $(\mu \mathrm{W})$ over $<0.4 \mathrm{THz}$ bandwidth, allocated among a few, irregularly distributed modes of dissimilar intensities. This was a result of dual-cavity induced suppression of the multimode operation regime. ${ }^{21}$ Such coupled cavity architectures are extremely beneficial for generating ultrashort $\mathrm{THz}$ pulses through injection seeding. ${ }^{22}$ Using a very small intracavity spacing $(1.5-2 \mu \mathrm{m})$, and predefined coupled-cavity lengths, effective chromatic dispersion compensation was achieved. However, this was only possible over a very limited frequency range, due to the nonlinear phase-frequency relation associated with the dispersion. Oscillation in the group delay dispersion (GDD) was expected, as the phase of the reflected light, and GDD, change periodically with the optical frequency, owing to resonance effects. ${ }^{22}$

Despite these advances, there remains a lack of miniaturized technologies for high-power $(>5 \mathrm{~mW})$, broadband $(\sim 1 \mathrm{THz}$ bandwidth) THz QCL FCSs, providing phase-locked, evenly spaced, lines of comparable intensity over the entire laser dynamic range. Furthermore, for high-precision $\left(10^{-11}\right)$ and high-sensitivity (one part in $10^{-6} \mathrm{~cm}^{-1} / \mathrm{Hz}^{-1 / 2}$ ) metrological applications, there is a further requirement for fine frequency tunability of the comb lines over a broad spectral window, combined with an (ideally) zero time jitter of the phase-locked modes, and small phase fluctuations. This would allow $\mathrm{THz}$ QCL FCSs to be fully stabilized against primary frequency standards. $^{24}$

Here, we present a record dynamic range THz QCL FCS based on a monolithic coupled-cavity architecture, comprising a heterogeneous THz-frequency QCL, with a wide (3.2) dynamic range, and an on-chip solution-processed multilayer graphene saturable absorber (GSA) reflector, and demonstrate stable injection locking. The gapless nature of graphene, and the related frequency-independent absorption, ultrafast recovery time, ${ }^{25}$ low saturation fluence, ${ }^{26}$ and ease of fabrication ${ }^{27}$ and integration, ${ }^{28,29}$ makes it an appealing nonlinear optical component in the infrared, and ideal for developing THz QCL FCSs, with performances far-beyond any alternative technology developed so far. Furthermore, graphene can be employed to introduce intensity dependent losses into the external laser cavity. $^{30,31}$ To date, graphene has been employed as external element, in transmission, to induce changes in the emission spectra of a THz QCL emitting over a limited $(0.15 \mathrm{THz})$ spectral window. $^{32}$

Here, we use a heterogeneous $17-\mu$ m-thick GaAs/AlGaAs heterostructure comprising three active modules, with gain bandwidths centered at $2.5,3$, and $3.5 \mathrm{THz}$ and comparable threshold current densities $\left(J_{\text {th }}\right)$. This leads to a very broad operational dynamic range of the heterogeneous gain medium: $J_{\mathrm{dr}}=J_{\max } / J_{\text {th }}=3.2$, where $J_{\max }$ is the maximum working current density. ${ }^{15,17}$ Sample fabrication is based on a metal-metal processing technique, with lossy side absorbers lithographically implemented along the waveguide edges, as discussed in Methods. ${ }^{15,33}$ The resulting lasers work as fully stabilized optical frequency comb synthesizers over $16 \%$ of their dynamic range, ${ }^{15}$ as we recently demonstrated through the full-control and stabilization of the characteristic FCS parameters, i.e., the 

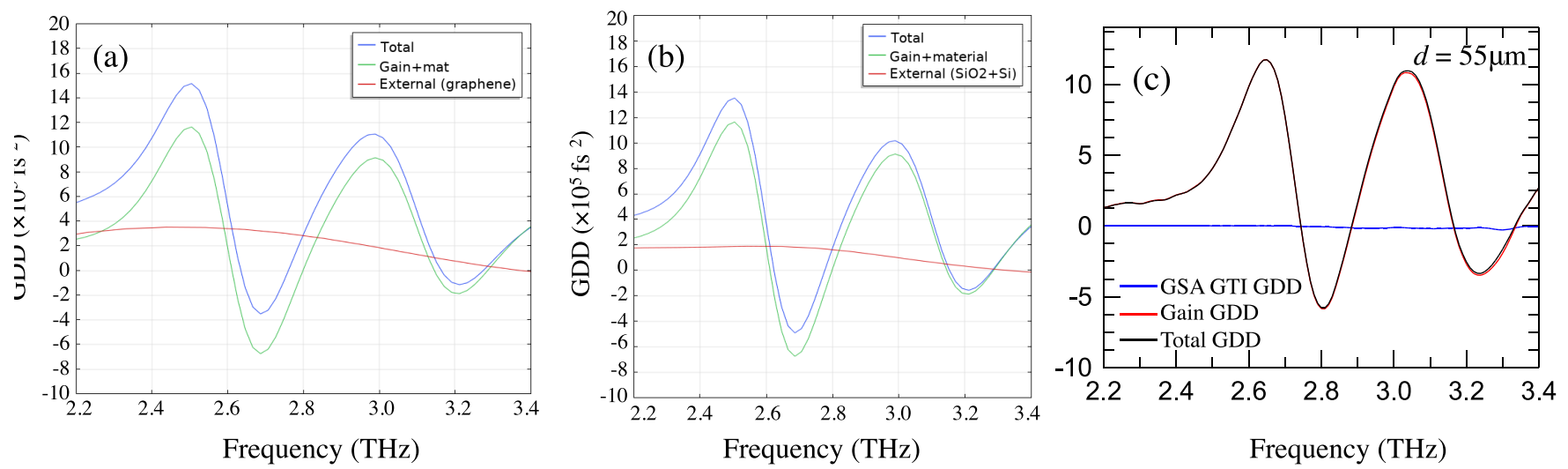

Figure 2. (a) Individual simulated group delay dispersion (GDD) of the GTI composed by GSA reflector, the QCL comb material and gain GDD,

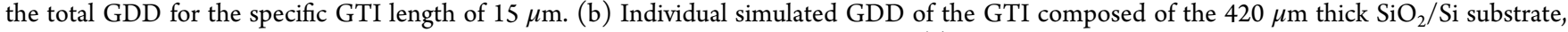
the QCL comb material and gain GDD, the total GDD for a GTI length of $15 \mu \mathrm{m}$. (c) Individual simulated GDD of the GTI composed by the GSA reflector, the QCL comb material and gain GDD, the total GDD for the specific GTI length of $55 \mu$ m, i.e., for an "on-resonance GTI".
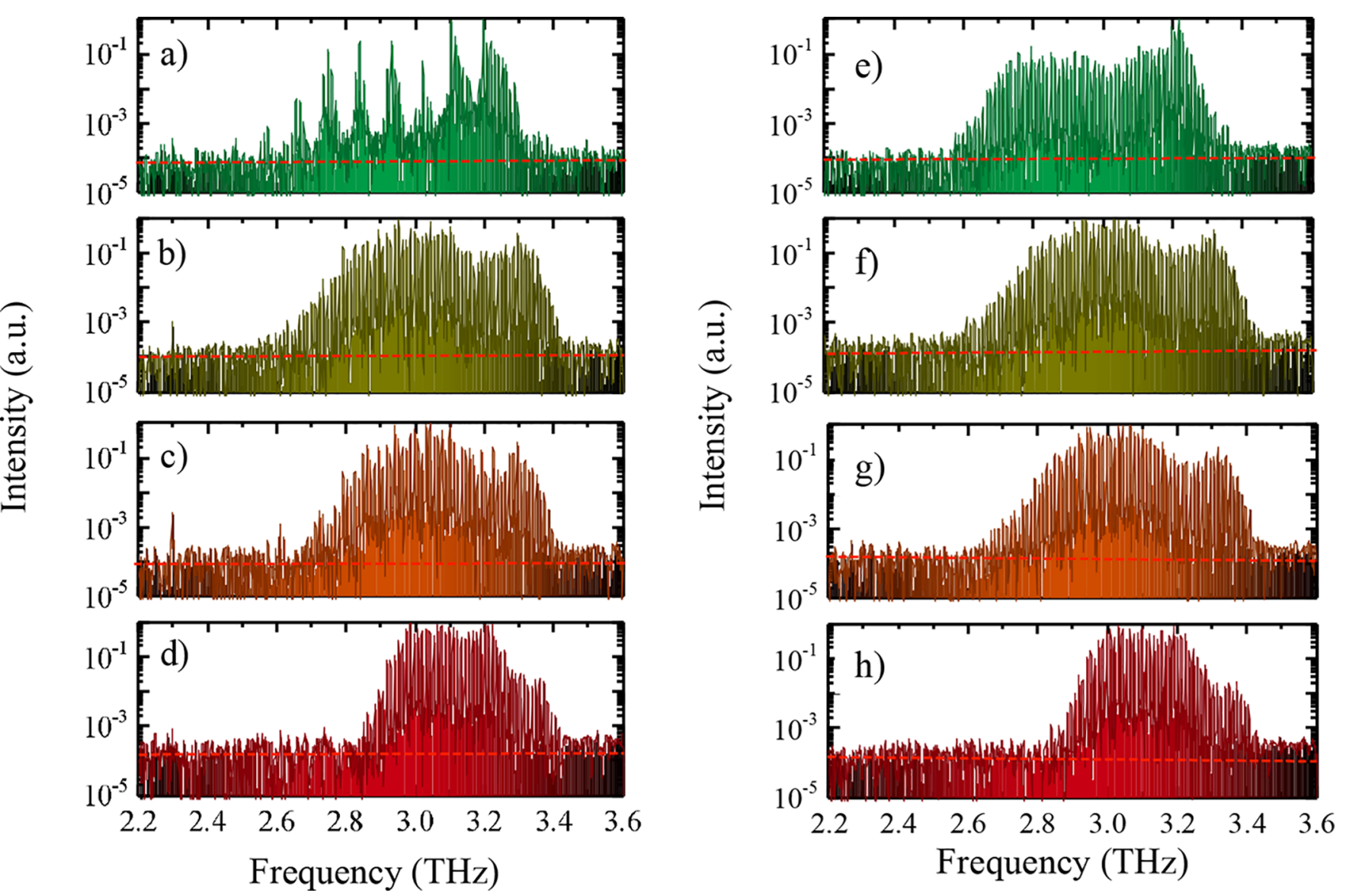

Figure 3. FTIR emission spectra collected under vacuum with a Bruker (Vertex 80) spectrometer in rapid scan mode, with a $0.075 \mathrm{~cm}^{-1}$ resolution in the $G$ sample $(\mathrm{a}-\mathrm{d})$ and in the $R$ sample $(\mathrm{e}-\mathrm{h})$. The $\mathrm{QCL}$ is operated in $\mathrm{CW}$ at a fixed heat sink temperature $=15 \mathrm{~K}$ at driving currents of $(\mathrm{a}, \mathrm{e})$ $560 \mathrm{~mA},(\mathrm{~b}, \mathrm{f}) 880 \mathrm{~mA},(\mathrm{c}, \mathrm{g}) 950 \mathrm{~mA}$, and $(\mathrm{d}, \mathrm{h}) 1050 \mathrm{~mA}$, respectively. The dashed horizontal lines indicate the noise floor of the measurements.

separation between adjacent modes and the carrier offset frequency, measured using a multiheterodyne detection scheme, in which the QCL-comb was mixed with a fully stabilized optically rectified $\mathrm{THz}$-frequency comb. ${ }^{24}$

The graphene reflector is prepared by liquid phase exfoliation (LPE) of graphite in a water/surfactant solution (see Methods). ${ }^{30}$ The resulting film is $\sim 65 \mathrm{~nm}$ thick, as determined by atomic force microscopy ${ }^{30}$ and covers a surface of about $1 \mathrm{~cm}^{2}$. Raman spectroscopy is used to monitor the quality of the flakes at each step of the preparation process as well as to qualitatively estimate, in combination with electrical transport tests, the Fermi energy, $E_{\mathrm{F}} \leq 250 \mathrm{meV} .^{30}$ The resultant reflector $(58 \% \text { reflectivity in the } \mathrm{THz})^{30}$ behaves as an intraband driven (fast) saturable absorber at $\mathrm{THz}$ frequencies, providing $80 \%$ transparency modulation, as a result of the intraband induced absorption bleaching. ${ }^{30}$ This is confirmed when using our fully stabilized optical frequency 
a)

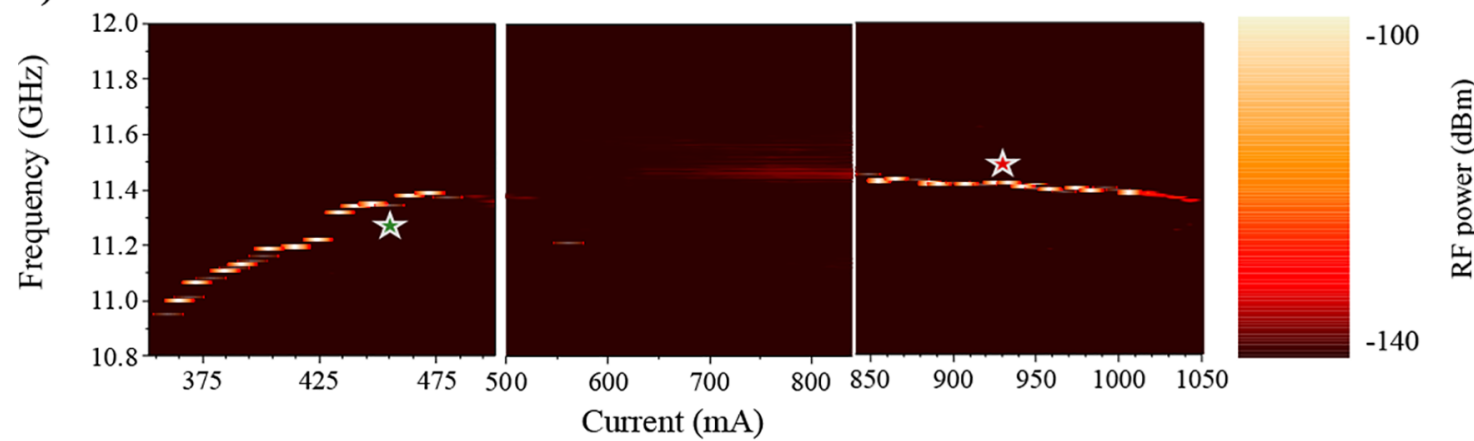

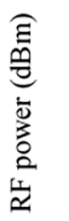
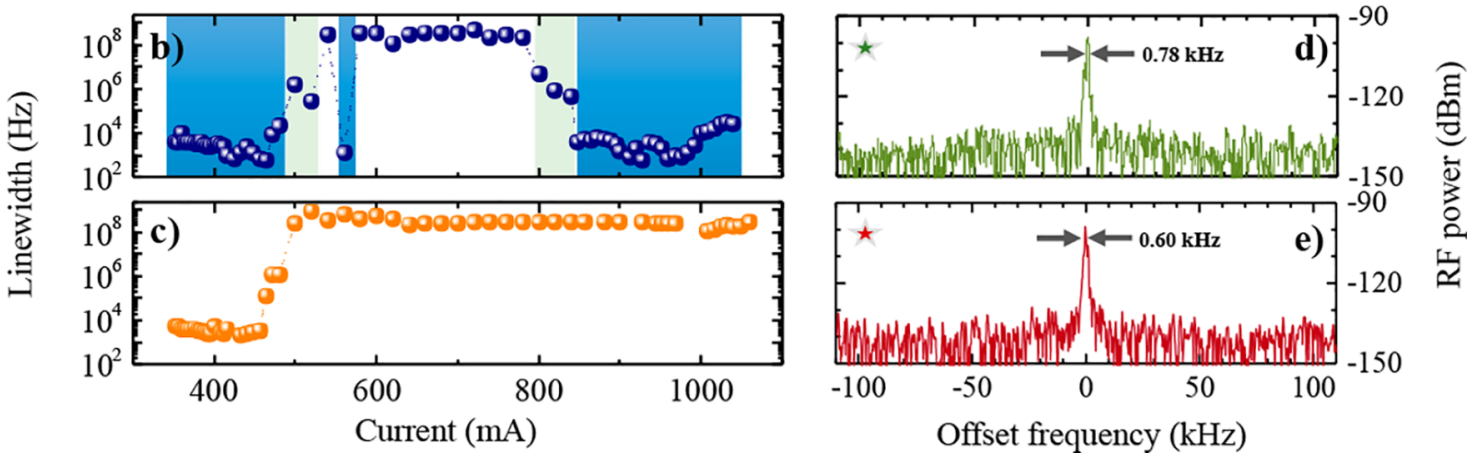

Figure 4. Analysis of intermode beatnote. (a) Intermode beatnote map as a function of QCL bias current for a $3.6 \mathrm{~mm}$ long, $50 \mu \mathrm{m}$ wide laser bar integrated on-chip with a GSA reflector. The beatnote signal is extracted from the bias line with a bias-tee and recorded with an RF spectrum analyzer (Rohde and Schwarz FSW; RBW: $500 \mathrm{~Hz}$, video bandwidth (VBW): $500 \mathrm{~Hz}$, sweep time (SWT): 20 ms, RMS acquisition mode). All measurements are performed in $\mathrm{CW}$ at a fixed heat sink temperature $=15 \mathrm{~K}$. The two star symbols indicate representative driving current regimes $(I=456 \mathrm{~mA})$ and $(I=928 \mathrm{~mA})$ in which the device behaves like a stable frequency comb synthesizer. $(\mathrm{b}, \mathrm{c})$ Intermode beatnote line width as a function of driving current measured on (b) the GSA coupled QCL ( $G$ sample) and (c) the bare laser ( $R$ sample); (d,e) Intermode beatnote trace recorded at (c) $I=456 \mathrm{~mA}$ with center frequency at $11.345 \mathrm{GHz}$, and (d) at $I=928 \mathrm{~mA}$ with center frequency at $11.426 \mathrm{GHz}$, respectively.

comb in pulsed mode ( $10 \%$ duty cycle) (see Supporting Information and Supplementary Figure S1).

The graphene reflector is then mounted on a $\mathrm{Cu}$ mount, via a thin indium foil (see Figure 1a), to ensure optimal thermal contact with the QCL Cu mount, with which it is in close thermal contact.

The graphene reflector is coupled on-chip, perpendicular to the back facet of a 3.6-mm-long, 50- $\mu \mathrm{m}$-wide heterogeneous $\mathrm{THz}$ QCL, with a separation of $15 \mu \mathrm{m}$, meaning that it can be approximated as part of the laser cavity, and finely aligned by means of calibrated screws. A picture of the GSA reflector is shown in the bottom part of Figure 1a. The unbiased reflector and the QCL, forming a coupled cavity with a $15 \mu \mathrm{m}$ air gap (GTI), are then mounted onto the coldfinger of a continuousflow He cryostat. Light emission (Figure $1 \mathrm{~b}$ ) is measured from the QCL using the facet opposite to the graphene reflector, as illustrated in the graphics of Figure 1a. This chip-scale embedded integration of the GSA ensures long-term stability, appropriate thermal management, and reproducibility of the experimental results.

In this configuration, the Gires Tournois interferometer does not affect the total QCL GDD, as shown by numerical simulations of the dispersion compensation performed using a finite element method (Comsol Multiphysics) (see Methods), Figure 2a-c.

At a distance of $15 \mu \mathrm{m}$, the total GDD does not show any significant variation, either when the QCL is integrated with the GSA reflector (Figure 2a), or when it is combined with the bare $420 \mu \mathrm{m}$ thick $\mathrm{SiO}_{2} / \mathrm{Si}$ substrate (Figure $2 \mathrm{~b}$ ). This is expected, as the phase change introduced by the GTI is almost negligible for such a small distance. Therefore, in this strong coupling condition, it is expected that dispersion compensation does not play any role in the phase-dynamics of the QCL comb modes, that are, in practice, unperturbed by the GTI, as in the case when a $\mathrm{SiO}_{2} / \mathrm{Si}$ substrate is strongly coupled to the QCL. We then perform the same simulations while matching the distance $d$ with the first resonance of our GTI, ${ }^{23}$ i.e., $d=55$ $\mu \mathrm{m}$ (Figure 2c). Unlike what obtained with a coupled Au metal mirror, ${ }^{23}$ the GDD introduced by the GTI is over 1 order of magnitude lower than that arising from the QCL gain, meaning that no dispersion compensation is expected. We consider this effect to be a result of the high absorption of the graphene stack, which limits the feedback to the QCL.

In the following the GSA coupled-cavity laser is labeled as "G", with "R" referring to the same QCL with the on-chip GSA reflector.

Comparison between continuous-wave (CW) currentvoltage (I-V) and light-current $(\mathrm{L}-\mathrm{I})$ characteristics (Figure 1b) of the GSA coupled cavity laser (sample G) and of the bare, QCL (sample R) shows that, while the device transport is not affected by the presence of the coupled reflector, the optical power benefits from the nonlinear reflector. An optical power of $8 \mathrm{~mW}$ is measured from the front facet of sample G, a factor of 2 larger than that of the bare sample R. Furthermore, the GSA reflector leads to factor $\sim 2.3$ increase in slope efficiency.

Considering the $\pm 40^{\circ}$ divergence (Supplementary Figure S2) measured from our double-metal THz QCL comb, and since the power emitted from the back facet of the QCL is initially equal to that measured from the reference sample $R$, 


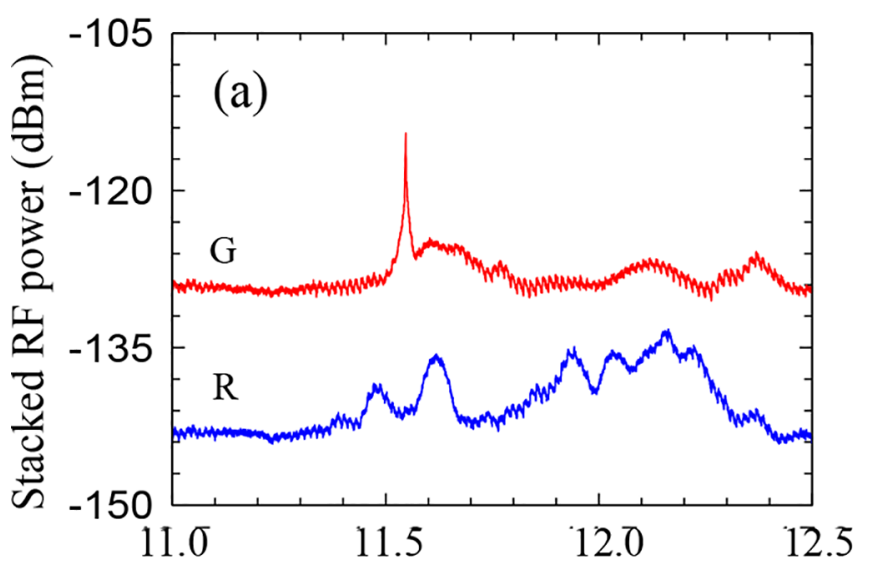

Intermode beatnote frequency

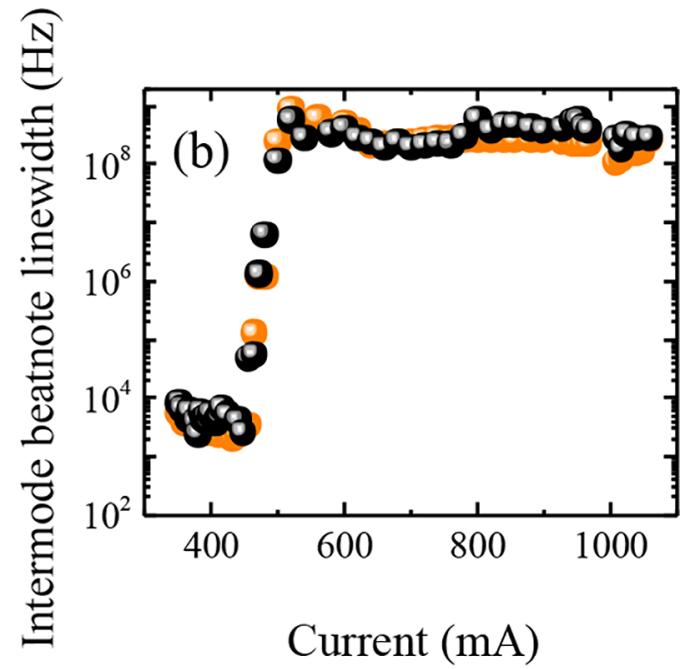

Figure 5. (a) Stacked intermode beatnote traces recorded at a heat sink temperature $=15 \mathrm{~K}$, while driving the GSA-coupled QCL (G) and the bare laser (R) at $J=350 \mathrm{~A} / \mathrm{cm}^{2}$. The spectrum analyzer settings are RWB: $10 \mathrm{kHz}$, VBW: $100 \mathrm{kHz}$, and SWT: $500 \mathrm{~ms}$. The line width is limited by the RBW of the spectrum analyzer. (b) Intermode beatnote line width as a function of driving current measured on the bare laser, while coupling it with the $420 \mu \mathrm{m}$ thick $\mathrm{SiO}_{2} / \mathrm{Si}$ substrate, placed at a distance of $15 \mu \mathrm{m}$ from the QCL back facet.

the intensity of the $\mathrm{THz}$ beam impinging on the GSA reflector varies from $I_{0} \sim 100 \mathrm{~W} / \mathrm{cm}^{2}$ just above threshold $(I=400 \mathrm{~mA})$ to $I_{0} \sim 800 \mathrm{~W} / \mathrm{cm}^{2}$ at the optical peak power $(I=835 \mathrm{~mA})$, well above the saturation intensity $\left(I_{s} \sim 6.3-6.7 \mathrm{~W} / \mathrm{cm}^{2}\right)$ (ref 30 and Supporting Information) over the full dynamic range of the laser, confirming that the graphene film behaves as a saturable absorber. ${ }^{30}$

Representative CW Fourier transform infrared spectra (FTIR) under-vacuum (Figure $3 a-d$ ), measured for different currents in the $G$ sample, show that well above threshold $(>880 \mathrm{~mA})$ (Figure $3 \mathrm{~b}-\mathrm{d}$ ) the GSA reflector does not induce major changes in the spectral behavior of the laser (see Figure $3 \mathrm{f}-\mathrm{h}$ for comparison with the $\mathrm{R}$ sample) over most of its operational range. It activates some additional peaks in the 2.3-2.4 $\mathrm{THz}$ region (Figure $3 \mathrm{a}-\mathrm{d}$ ) that persist over the whole operational range, and that are absent in the bare laser (Figure $3 \mathrm{e}-\mathrm{h})$.

A remarkable difference is detected at $560 \mathrm{~mA}(J=311 \mathrm{~A} /$ $\mathrm{cm}^{2}$ ). The $\mathrm{G}$ sample spectrum in Figure $3 \mathrm{a}$ has families of lasing modes individually spaced by the cavity round trip time, but the families are separated by a frequency matching a high order harmonic (9th) of the cavity's round trip frequency. Such a phenomenon is typical of harmonic mode locking, happening when multiple pulses per round trip are generated as a consequence of the modulation applied at harmonics of the cavity's fundamental round trip frequency. ${ }^{34}$ The spectrum of the $\mathrm{G}$ sample then broadens gradually with bias, reaching a continuous bandwidth of $0.94 \mathrm{THz}(2.55-3.49 \mathrm{THz})$ at a current of $880 \mathrm{~mA}$, and a discontinuous bandwidth of 1.25 $\mathrm{THz}(2.30-3.55 \mathrm{THz})$, with $8 \mathrm{~mW}$ of $\mathrm{CW}$ power, distributed among 90 equally spaced optical modes, as shown in Figure $3 \mathrm{~b}$.

Figure $4 \mathrm{a}$ plots the corresponding free running electrical beatnote map, typically performed to characterize the coherence of the spectral emission of QCL-based frequency combs. ${ }^{13}$

Beatnote signals are extracted from the bias line, using a bias-tee, and recorded with a radio frequency (RF) spectrum analyzer. A change in the intermode beatnote signal map is seen for the whole operational range of the QCL, when compared to that of the reference laser. ${ }^{15,24}$ At $J=228 \mathrm{~A} / \mathrm{cm}^{2}$, when band alignment is fully reached, as typical of most QCL based frequency combs, the optical modes of the reference laser (sample R) lose their phase coherence and the intermode beatnote significantly broadens ( $>100 \mathrm{MHz}$ line width) as the GVD becomes large enough to prevent FWM from locking the lasing modes in frequency and phase simultaneously. ${ }^{15,24}$ This contrasts with the bias-dependent evolution of the beatnote map of the G sample (Figure 4a). The introduction of the GSA reflector causes a significant enlargement (from 16\% (Figure $4 \mathrm{c}$, and ref 15 ) to $55 \%$ of the laser operational range (Figure $4 a)$ ) of the dynamic range in which $\mathrm{THz}$ QCL frequency comb is observed.

The comparative analysis of the beatnote line width (Figure $4 \mathrm{~b}, \mathrm{c}$ ) discloses the efficacy of our approach. A set of individual beatnotes, as narrow as $780 \mathrm{~Hz}$ (Figure 4d), persists in the current range between 350 and $480 \mathrm{~mA}$, as shown in Figure $4 \mathrm{~b}$. The beatnote is a factor of 5 narrower than measured in the reference sample, ${ }^{24}$ (Figure 4c), at specific biases/currents, thus suggesting that the GSA integration improves the phase locking of the optical modes. In analogy with the bare laser (sample R), ${ }^{15,24}$ in the region between 350 and $480 \mathrm{~mA}$, the RF beatnote signals are blue-shifted with a coefficient 3.6 $\mathrm{MHz} / \mathrm{mA}$. This is a consequence of the changes in the relative distance between the beating modes, induced by the chromatic dispersion in the gain spectrum.

When the current in sample $G$ is increased from 480 to 500 $\mathrm{mA}$, the single beatnote line width increases to $10 \mathrm{kHz}$, although it still preserves its narrow nature (Figure 4b). Conversely, above $476 \mathrm{~mA}$, the bare laser (sample R) (Figure $4 c)^{15}$ looses its phase coherence ${ }^{24}$ and develops a broad beatnote regime, reaching $\sim 300 \mathrm{MHz}$ line width at $500 \mathrm{~mA}$.

In the current range $500-525 \mathrm{~mA}$, the beatnote of sample $\mathrm{G}$ remains single, but superimposed to a broader beatnote (100$880 \mathrm{kHz})$. Over a very small $(10 \mathrm{~mA})$ current range, around $570 \mathrm{~mA}$, the beatnote turns again single and narrow $(950 \mathrm{~Hz})$. This is a signature that the dispersion compensation in sample $\mathrm{G}$ enables phase locking of the emitted optical modes.

In the 580-780 $\mathrm{mA}$ range, the beatnote line width increases to $200-500 \mathrm{MHz}$, signature of a transport regime dominated by dispersion. However, a single beatnote is still visible, 

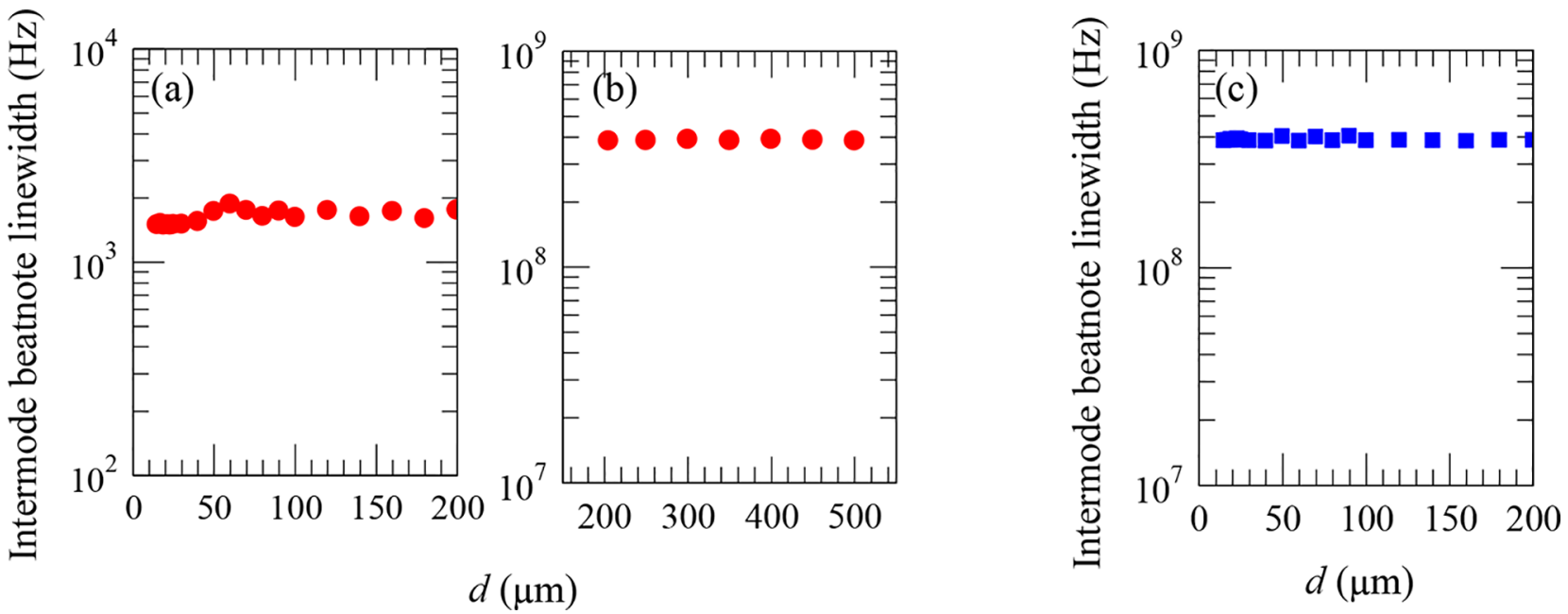

Figure 6. $(\mathrm{a}, \mathrm{b})$ Evolution of electrical intermode beatnote line width of GSA-coupled QCL as a function of the distance d of the GSA from the laser facet, for a fixed driving current of $900 \mathrm{~mA}$. (c) Evolution of the electrical intermode beatnote line width of the QCL coupled with a 420- $\mu \mathrm{m}$-thick $\mathrm{SiO}_{2} / \mathrm{Si}$ substrate, as a function of the distance $\mathrm{d}$ of the $\mathrm{SiO}_{2} / \mathrm{Si}$ substrate from the laser facet, for a fixed driving current of $900 \mathrm{~mA}$. All measurements are performed in $\mathrm{CW}$ at $15 \mathrm{~K}$. The beat-note signal is extracted from a bias line, employing a bias-tee connected to a RF optical spectrum analyzer (OSA) in RMS acquisition mode (OSA setting: RWB: $500 \mathrm{~Hz}, \mathrm{VBW}: 500 \mathrm{~Hz}, \mathrm{SWT}: 50 \mathrm{~ms}$ ).

superimposed on a broader signal (see Figure 5a). Although wider, the presence of such an individual beatnote enables locking it to a microwave reference, in order to mode-lock the QCL and, consequently, reduce the beatnote line width. ${ }^{35}$

Similarly, in the 780-835 $\mathrm{mA}$ range, the beatnote remains single but turns narrower and is superimposed on a broader one $(100-790 \mathrm{kHz})$. In contrast, a fully dispersion dominated regime is observed in sample $\mathrm{R}$, over $>80 \%$ of its dynamic range (Figure 4c), ${ }^{15}$ i.e., for all currents $>536 \mathrm{~mA}$.

The most significant effect of the GSA reflector appears at driving currents $>845 \mathrm{~mA}$ (i.e., for $J=2.6-3.2 J_{\mathrm{th}}$ ), where the single beatnote line width preserves its narrow nature $(600$ $\mathrm{Hz}-10 \mathrm{kHz}$ ) (Figure $4 \mathrm{~b}, \mathrm{e}$ ). This corresponds to the highphase noise regime typically recorded at larger current densities in the bare laser (sample R), and in all other $\mathrm{THz}$ QCL combs, reported to date, when $J>1.16 J_{\text {th }}$.

This single and narrow beatnote is in contrast to that detected in the $\mathrm{R}$ sample, in which spontaneous FWM phenomena are typically unable to support dispersion compensation. ${ }^{13,15,20}$ The GSA reflector provides a clear advantage with respect to any alternative saturable absorbers developed so far at $\mathrm{THz}$ frequencies such as n-doped semiconductors (GaAs, GaP, and $\mathrm{Ge}$ ). Although these materials can be used as $\mathrm{THz}$ SAs, they require electric fields of $10 \mathrm{~s} \mathrm{kV} / \mathrm{cm}$ and cannot be easily integrated with a THz QCL using an approach similar to that described here, without inducing a major detrimental increase of the external cavity losses due to free carrier absorption.

By progressively driving the laser toward the negative differential resistance regime, the narrow beatnote red-shifts with a coefficient $-0.33 \mathrm{MHz} / \mathrm{mA}$, mainly due to the local heating of the lattice at higher driving currents.

Figure 4d,e plots the intermode beatnote spectra in the two most interesting transport regimes in which in the QCL behave like a comb: $456 \mathrm{~mA}$ (Figure 4d) and $928 \mathrm{~mA}$ (Figure 4e). Beatnote line widths as narrow as $780 \mathrm{~Hz}$ (Figure 4d) and 600 $\mathrm{Hz}$ (Figure 4e) can be seen the narrowest to date in any $\mathrm{THz}$ QCL FCS, to the best of our knowledge. Correspondently, in the latter case, the spectrum shows a $0.94 \mathrm{THz}$ spectral coverage, with a high output power $(8 \mathrm{~mW}$ of $\mathrm{CW}$ power; 40
mW of peak power in pulsed mode, maximum wall plug efficiency $0.1 \%$ ), distributed among 90 equally spaced optical modes. This sets the state of the art THz QCL frequency combs. It is worth mentioning that the line width values are ultimately limited by the jitter of the beat-note, since the laser is not stabilized.

The explanation of the behavior at $\mathrm{I}>845 \mathrm{~mA}$ is a consequence of the mechanism through which the graphenerelated dynamics contributes to stabilize the QCL optical modes. Frequency and phase locking of the modes, prerequisite of comb formation, can be obtained through FWM generated by either fast saturable gain or loss in semiconductor lasers. ${ }^{36}$ The former mechanism leads to a frequency modulated output, while the latter is associated with amplitude modulation. In THz QCL FC, both frequency and amplitude modulation are typically present, ${ }^{37}$ and act simultaneously. In our setup, the gain and absorption are spatially separated, so they do not average out to a local net gain/loss, and create a spatially dependent profile within the cavity. The above arguments explain why, as a result of the interaction of the field emitted by the QCL with the inherently fast GSA, ${ }^{30}$ and the related reinjection of this field into the laser cavity, the fast saturable loss of the GSA contributes to the locking between the modes, which manifests itself by the observed extremely narrowed beatnote.

The dynamical processes behind the generation of such a sharp and narrow beatnote, following the above arguments, are then investigated by performing time-domain simulations based on the Maxwell-Bloch equations in the configuration in which the QCL is uncoupled (sample R) or tightly coupled (sample G) with the GSA. We use the mbsolve ${ }^{38}$ software, which is an open-source solver for the one-dimensional Maxwell-Bloch equations capable of handling spatial regions of different materials. We investigate the dynamical behavior of our experimental system, as described in the Supporting Information. The QCL is modeled as two-level gain medium, with the physical parameters summarized in Table I (Supporting Information). Subsequently, we derive the intermode beatnote spectrum from the optical field, for both cases. The simulation results (Figure S3), in agreement with 

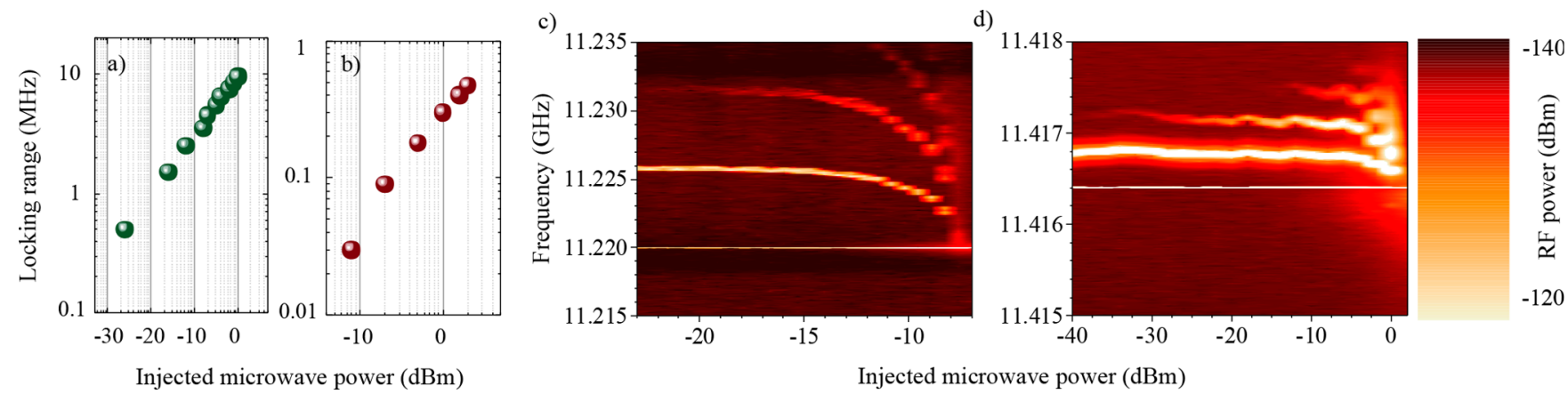

Figure 7. Coherent injection locking. (a,b) Locking range as a function of RF-power transmitted inside the cavity of a THz QCL-comb integrated on-chip with a GSA reflector. The RF and dc-bias signals are brought to the QCL using a $60 \mathrm{GHz}, 50 \Omega$ coplanar probe positioned at one end of the QCL ridge. The intermodal beatnote (IBN) frequency is at a repetition frequency $\sim 11 \mathrm{GHz}$, and is electrically extracted from the QCL by connecting the RF-synthesizer to the input port of a $40 \mathrm{GHz}$ directional coupler, with the output port of the coupler connected to the ac input of a $40 \mathrm{GHz}$ bias-Tee. The dc line of the bias-Tee is connected to the power supply that drives the QCL at a constant current of (a) $425 \mathrm{~mA}$, and (b) $980 \mathrm{~mA}$. The spectra are collected with the same settings of Figure 3a. (c,d) IBN spectra for different RF injected powers. The RF frequency is held constant at approximately (c) $6 \mathrm{MHz}$ or (d) $0.4 \mathrm{MHz}$ apart from the cavity round trip frequency, and the laser is operating at (c) $425 \mathrm{~mA}$, and (d) $980 \mathrm{~mA}$. Microwave spectra are on a logarithmic scale for different injected powers.

our experimental findings, show a reduction of the intermode beatnote line width when the QCL is integrated with the GSA.

In addition to the saturable absorption effect inside graphene, the reflector also features Fresnel reflection on the graphene surface. Since this component is spatially separated from the saturable absorption occurring inside graphene, it might contribute to frequency comb stabilization through the same mechanism as the fast saturable gain in the active region. These effects then try to force the QCL into frequencymodulated operation, while the saturable absorption in graphene helps regularize the remaining amplitude modulations.

The combination of the above effects can explain our experimental observations.

To further confirm our arguments, we perform a further set of experiments.

First, Figure $5 b$ compares the reference unperturbed QCL beatnote evolution with the corresponding beatnote line width evolution recorded when the $420-\mu \mathrm{m}$-thick $\mathrm{Si} / \mathrm{SiO}_{2}$ substrate is coupled (at $15 \mu \mathrm{m}$ distance) to one end of the QCL laser cavity, in the same position of the GSA reflector. No difference is seen in the beatnote map with respect to the bare laser, confirming that the dispersion compensation, arising from the $\mathrm{Si} / \mathrm{SiO}_{2} 50 \%$ reflector, as predicted by numerical simulations (Figure 2a,b), does not play a role, and the GSA reflector is driving the intermode dynamics.

Second, we perform two set of experiments in which we collect the intermode beatnote line widths by varying the distance of both GSA and $\mathrm{Si} / \mathrm{SiO}_{2}$ from the end of the QCL laser cavity $(d)$, respectively, while keeping the current at $I=$ $900 \mathrm{~mA}$, i.e., in the region in which the most significant differences are unveiled.

In both cases, we first vary $d$ finely in the $15-25 \mu \mathrm{m}$ range, then in the range $25-200 \mu \mathrm{m}$ with the impinging laser beam intensity $I \leq I_{s}$, and then in the range $200-500 \mu \mathrm{m}$, where $I \ll$ $I_{s}$. The rationale is that, since GTIs are extremely sensitive to the distance from the laser facet, any change of the beatnote map would be a signature of a GDD compensation operated by the GTI. The analysis of the intermode beatnote line width (Figure 6a,b) at $900 \mathrm{~mA}$, shows that in our G sample, by varying $d$ in and out resonance, the intermode beatnote remains single and preserves its narrow nature (Figure 6a) for $d \leq 200 \mu \mathrm{m}$, unlike what experimentally observed in the case of a dispersion compensated GTI THz QCL comb, ${ }^{23}$ achieved by coupling a metal mirror.

In the latter case, a narrow $(\sim 3 \mathrm{kHz})$ and intense beatnote is only achieved at periodic positions of the external metal coupling element. ${ }^{23}$ For $d>205 \mu \mathrm{m}$ (Figure 6b), the GSAintegrated QCL behavior is identical to that of the bare laser, a signature that the GSA plays no role. As expected, in the $\mathrm{Si} /$ $\mathrm{SiO}_{2}$-coupled QCL, the distance does not affect the broad line width $(>100 \mathrm{MHz})$, which persists over the whole range of distances (Figure 6c).

The ability to coherently frequency stabilize the GSA reflector is further proved by applying a direct RF modulation at its round-trip frequency. Injection of a periodic signal into an oscillator is commonly used to stabilize emission frequency and/or laser cavity modes separation, ${ }^{39}$ and corresponds to round-trip frequency stabilization, as normally achieved by injection of an external microwave signal on the driving current. Reference 35 demonstrated injection locking of the intermode frequency difference of $\mathrm{THz}$ QCL over hundreds of $\mathrm{MHz}$ by driving the laser bias with a microwave signal close to this frequency. Reference 40 achieved the stabilization of the frequency difference between two lateral modes of a $\mathrm{THz} \mathrm{QCL}$ with corresponding frequency locked line widths $\leq 10 \mathrm{~Hz}$ and with a negligible drift. Here, we demonstrate the stability of our GSA-coupled QCL-comb intermode separation by measuring the shape of its photocurrent spectrum in the microwave range, around the cavity round-trip frequency, where a sharp single peak proves stable phase and frequency relation between adjacent laser modes.

Figure 7a,b shows all-electrical injection locking of a narrow beatnote to the RF oscillator, and the corresponding locking range, as a function of the RF-power transmitted inside the cavity of sample $G$. This follows the square root behavior predicted by Adler's equation. ${ }^{35}$

The RF-signals capture the comb oscillator, imposing an oscillation frequency even when the RF power is as low as -29 $\mathrm{dBm}$ (Figure $7 \mathrm{a}, I=400 \mathrm{~mA}$ ), provided that the two oscillators are at sufficiently close frequencies and their coupling is strong enough. Equally, with a higher laser current (Figure 7b, 980 $\mathrm{mA}$ ), locking is observed, albeit with higher injection power levels. $^{39}$ 
To investigate the injection locking dynamics further, we retrieve the beat note spectra, measured in the single beatnote regimes, as the injected RF power is increased. The broad beatnote is pulled toward the frequency of the injected signal $(11.2200 \mathrm{GHz}$, Figure $7 \mathrm{c} ; 11.4164 \mathrm{GHz}$, Figure $7 \mathrm{~d}$ ) and finally locked, as shown in Figure $4 \mathrm{c}, \mathrm{d}$ at the different bias currents of $425 \mathrm{~mA}$ (Figure 7c) and $980 \mathrm{~mA}$ (Figure 7d), respectively. Two side peaks $\sim 9 \mathrm{dBm}$ weaker than the initial beatnote remain for RF injected powers up to $-8 \mathrm{dBm}$ in Figure $7 \mathrm{c}$, then vanish, and the microwave spectrum of the intermode beating is fully controlled by the injected signal. The noise floor around the locked narrow beatnote is $\sim 20 \mathrm{~dB}$, i.e., weaker than the peak power of the originally broad beatnote. This proves that the intermode beatnote power is almost completely locked.

In conclusion, we demonstrated that the free-running phase coherence of broadband $\mathrm{THz}$ QCLs with a heterogeneous active region can be enhanced by on-chip coupling to a saturable absorber prepared from a liquid phase exfoliated graphite film. With the on-chip integration of a GSA reflector on a fully stabilized $\mathrm{THz}$ comb, operating only over $16 \%$ of its dynamic range, ${ }^{24,15}$ we achieve stable comb operation through $55 \%$ of the laser operational range, with a beatnote line width as narrow as $600 \mathrm{~Hz}, 8 \mathrm{~mW}$ of $\mathrm{CW}$ power and 90 equally spaced optical modes covering a $0.94 \mathrm{THz}$ spectral bandwidth, with more than three-decade reduction of phase-noise over an additional $15 \%$ of this range. We also achieve injection locking over the same operational range as the FCS, showing the stability of the comb operation. Our compact and frequency agile design, together with the high optical power, the large number of optical modes, and the narrow beatnote line width, well suited for dual-comb $\mathrm{THz}$ spectroscopy ${ }^{41}$ and hyperspectral imaging, ${ }^{42}$ paves the way to deliver an all-in-one miniaturized nanoengineered frequency comb electrical sources for spectroscopic and nanoscale applications in the far-infrared. Our design, in conjunction with graphene's fast recovery time and saturation fluence, could produce time domain pulses with sub-ps widths and average power comparable to the CW operation level of a QCL.

\section{METHODS}

QCL Fabrication. Lasers are fabricated in a metal-metal waveguide configuration. We first perform $\mathrm{Au}-\mathrm{Au}$ thermocompression wafer bonding of the $17-\mu \mathrm{m}$-thick active region (sample L1458) onto a highly doped GaAs substrate, followed by the removal, through a combination of mechanical lapping and wet etching, of the host GaAs substrate of the molecular beam epitaxial (MBE) material. The $\mathrm{Al}_{0.5} \mathrm{Ga}_{0.5}$ As etch stop layer is removed using HF etching. Laser bars are then defined by inductively coupled plasma etching, which leads to almost vertical sidewalls (hence uniform current injection into the full gain region). Following etching, a $\mathrm{Cr} / \mathrm{Au}(10 \mu \mathrm{m} / 150 \mu \mathrm{m})$ top contact is deposited along the center of the ridge surface, leaving a thin region uncovered along the ridge edges. The 5 $\mu$ m-wide $\mathrm{Ni}$ (5-nm-thick) side absorbers are then deposited over the uncovered region, using a combination of optical lithography and thermal evaporation. Such lossy side absorbers are intended to inhibit lasing of the higher order lateral modes by increasing their threshold gain. ${ }^{5}$ Finally, the backside of the substrate is lapped down to $150 \mu \mathrm{m}$ to optimize thermal management and enable operation in CW. Laser bars, $50 \mu \mathrm{m}$ wide and $3.6 \mathrm{~mm}$ long, are then cleaved and mounted on a Au- coated $\mathrm{Cu}$ bar, and then onto the cold finger of a $\mathrm{He}$ continuous-flow cryostat.

Preparation of the Graphene Reflector. A water-based ink is prepared by ultrasonicating (Fisherbrand FB15069, Max power $800 \mathrm{~W}$ ) graphite flakes (Sigma-Aldrich) for $9 \mathrm{~h}$ in deionized water with sodium deoxycholate (SDC, $9 \mathrm{mg} \mathrm{mL}^{-1}$ ). This is then vacuum filtered using $100 \mathrm{~nm}$ pore-size nitrocellulose filters. This blocks the flakes, while water passes through, leading to a film on the surface of the filter. This is then placed on a $420-\mu$ m-thick intrinsic high-resistivity $\mathrm{Si} / \mathrm{SiO}_{2}$ double polished wafer (acting as a back reflection mirror) and annealed at $\sim 80{ }^{\circ} \mathrm{C}$ for $2 \mathrm{~h}$, to improve adhesion, followed by dissolution of the filter in acetone overnight.

Simulation of the Total GDD of the $\boldsymbol{G}$ sample. Numerical GDD simulations (Figure S2(a),(b)) are performed using a finite element method (Comsol Multiphysics). The simulated structure includes the end of the QCL waveguide and a $65 \mathrm{~nm}$ thick GSA on a $420 \mu \mathrm{m}$ thick $\mathrm{SiO}_{2} / \mathrm{Si}$ mirror, separated by $15 \mu \mathrm{m}$ from the laser back facet. This structure is then surrounded at a distance of a few $\lambda$ by vacuum and absorbing boundary conditions. As the $\mathrm{SiO}_{2}$ layer is very thick $(\sim 300 \mu \mathrm{m})$ compared to the separation between the GSA and the QCL facet, the amount of radiation that could be reflected back to the QCL from the $\mathrm{SiO}_{2} / \mathrm{Si}$ boundary is negligible. Thus, absorbing boundary conditions are set $\sim 100 \mu \mathrm{m}$ inside the $\mathrm{SiO}_{2}$, as well as at all the other external boundaries of the simulation domain. The imaginary part of the refractive index of graphene is computed from the experimental values of the absorption coefficients reported in ref 30 , while the real part is numerically computed considering a graphene layer having a total scattering time $\tau=0.1 \mathrm{ps}$ and a chemical potential $\mu_{\mathrm{F}}=$ $250 \mathrm{meV}$. The variation of the imaginary part under illumination from the QCL is computed according to the expected reduction of the saturable absorption coefficient. The variation of the real part is then computed applying the Kramers-Krönig equations.

$\mathrm{THz}$ radiation is injected into the QCL waveguide (from the end opposite to the GTI) and then reflected back into the QCL waveguide by the GTI. This allows us to obtain the amplitude and phase of the $S_{11}$ scattering parameter. ${ }^{2,23}$ The dispersion provided by the structure is computed from the second derivative of the phase. The final GDD profile takes into account the contributions from the semiconductor material and gain of the QCL, ${ }^{23}$ and that of the GTI. The frequency dependent refractive index of the material is computed using a Drude-Lorentz model, adding its deviation due to the QCL's gain, obtained from the Kramers-Kronig equations. The waveguide dispersion contribution, negligible with respect to the other terms, is neglected

All-Electrical Injection Locking of a Narrow Beatnote to an RF Oscillator. The modulation signal is supplied by an external stabilized RF synthesizer (Rohde \& Schwarz SMA100B) through a Bias-Tee (Tektronix PSPL5544). The beatnote signal is then extracted from the bias line using the same bias-tee, and recorded with an RF spectrum analyzer (Rohde \& Schwarz FSW). For this, a high-speed sub-miniature push-on (SMP) connector with an integrated coplanar transmission line waveguide is used, allowing high-frequency electrical signals to pass for fast electrical modulation, suppression of losses, and deformation at the wire bonding points. The electrical connections between one end of the QCL ridge and the coplanar probe are realized using short and thin $(20 \mu \mathrm{m})$ Au bonding wires. 


\section{ASSOCIATED CONTENT}

\section{(s) Supporting Information}

The Supporting Information is available free of charge at https://pubs.acs.org/doi/10.1021/acsphotonics.0c01523.

Additional information including Figures S1-S3, Table I, and equations (PDF)

\section{AUTHOR INFORMATION}

\section{Corresponding Author}

Miriam S. Vitiello - NEST, CNR - Istituto Nanoscienze and Scuola Normale Superiore, 56127 Pisa, Italy; ○ orcid.org/ 0000-0002-4914-0421; Email: miriam.vitiello@sns.it

\section{Authors}

Francesco P. Mezzapesa - NEST, CNR - Istituto Nanoscienze and Scuola Normale Superiore, 56127 Pisa, Italy

Katia Garrasi - NEST, CNR - Istituto Nanoscienze and Scuola Normale Superiore, 56127 Pisa, Italy

Johannes Schmidt - NEST, CNR - Istituto Nanoscienze and Scuola Normale Superiore, 56127 Pisa, Italy

Luca Salemi - NEST, CNR - Istituto Nanoscienze and Scuola Normale Superiore, 56127 Pisa, Italy

Valentino Pistore - NEST, CNR - Istituto Nanoscienze and Scuola Normale Superiore, 56127 Pisa, Italy

Lianhe Li - School of Electronic and Electrical Engineering, University of Leeds, Leeds LS2 9JT, U.K.; (1) orcid.org/ 0000-0003-4998-7259

A. Giles Davies - School of Electronic and Electrical Engineering, University of Leeds, Leeds LS2 9JT, U.K.

Edmund H. Linfield - School of Electronic and Electrical Engineering, University of Leeds, Leeds LS2 9JT, U.K.

Michael Riesch - Department of Electrical and Computer Engineering, Technical University of Munich, 80333 Munich, DE, Germany; ○ orcid.org/0000-0002-4030-2818

Christian Jirauschek - Department of Electrical and Computer Engineering, Technical University of Munich, 80333 Munich, DE, Germany

Tian Carey - Cambridge Graphene Centre, University of Cambridge, Cambridge CB3 OFA, U.K.

Felice Torrisi - Cambridge Graphene Centre, University of

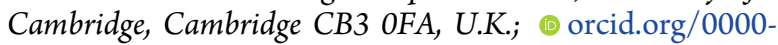
0002-6144-2916

Andrea C. Ferrari - Cambridge Graphene Centre, University of Cambridge, Cambridge CB3 OFA, U.K.; 이이.org/ 0000-0003-0907-9993

Complete contact information is available at:

https://pubs.acs.org/10.1021/acsphotonics.0c01523

\section{Notes}

The authors declare no competing financial interest.

\section{ACKNOWLEDGMENTS}

We acknowledge financial support from the ERC Project 681379 (SPRINT), the EU Graphene Flagship, ERC Grants Hetero2D, GSYNCOR, MINERGRACE; EPSRC Grants EP/ $\mathrm{K} 01711 \mathrm{X} / 1$, EP/K017144/1, EP/N010345/1, and EP/ L016087/1; and the EU Quantum Flagships. E.H.L. acknowledges support from the Royal Society and the Wolfson Foundation. The data that support the plots within this paper and other findings of this study are available from the corresponding author upon reasonable request.

\section{REFERENCES}

(1) Hänsch, T. W. Nobel lecture: passion for precision. Rev. Mod. Phys. 2006, 78, 1297-1309.

(2) Udem, T.; Holzwarth, R.; Hänsch, T. W. Optical frequency metrology. Nature 2002, 416, 233-237.

(3) Consolino, L.; et al. Phase-locking to a free-space terahertz comb for metrological-grade terahertz lasers. Nat. Commun. 2012, 3, 1040.

(4) Consolino, L.; Cappelli, F.; Siciliani de Cumis, M.; De Natale, P. QCL-based frequency metrology from the mid-infrared to the $\mathrm{THz}$ range: a review. Nanophotonics 2018, 8 (2), 181-204.

(5) Holzwarth, R.; et al. Optical frequency synthesizer for precision spectroscopy. Phys. Rev. Lett. 2000, 85, 2264-2267.

(6) Belkin, M.; Capasso, F. New frontiers in quantum cascade lasers: High performance room temperature terahertz sources. Phys. Scr. 2015, 90, 118002.

(7) Lee, A. W. M.; Kao, T.-Y.; Burghoff, D.; Hu, Q.; Reno, J. L. Terahertz tomography using quantum-cascade lasers. Opt. Lett. 2012, 37, 217-219.

(8) Hänsch, T. W.; Picqué, N. Laser Spectroscopy and Frequency Comb. J. Phys.: Conf. Ser. 2013, 467, 012001.

(9) Vitiello, M. S.; Scalari, G.; Williams, B.; De Natale, P. Quantum cascade lasers: 20 years of challenges. Opt. Express 2015, 23 (4), $5167-5182$

(10) Terahertz semiconductor-heterostructure laser. Nature 2002, 417 (6885), 156-159.

(11) Vitiello, M. S.; et al. Quantum limited frequency fluctuations in a terahertz laser. Nat. Photonics 2012, 6 (8), 525-528.

(12) Consolino, L.; et al. Spectral purity and tunability of terahertz quantum cascade laser sources based on intracavity difference frequency generation. Science. Advances 2017, 3, No. e1603317.

(13) Rösch, M.; Scalari, G.; Beck, M.; Faist, J. Octave-spanning semiconductor laser. Nat. Photonics 2015, 9, 42-47.

(14) Turčínková, D.; Scalari, G.; Castellano, F.; Amanti, M. I.; Beck, M.; Faist, J. Ultrabroadband heterogeneous quantum cascade laser emitting from 2.2 to $3.2 \mathrm{THz}$. Appl. Phys. Lett. 2011, 99, 191104.

(15) Garrasi, K.; et al. High dynamic range, heterogeneous, terahertz quantum cascade lasers featuring thermally tunable frequency comb operation over a broad current range. ACS Photonics 2019, 6, 73-78.

(16) Li, L.; et al. Terahertz quantum cascade lasers with $>1 \mathrm{~W}$ output powers. Electron. Lett. 2014, 50, 309-311.

(17) Li, L. H.; et al. Broadband heterogeneous terahertz frequency quantum cascade laser. Electron. Lett. 2018, 54, 1229-1231.

(18) Rösch, M.; et al. Heterogeneous terahertz quantum cascade lasers exceeding $1.9 \mathrm{THz}$ spectral bandwidth and featuring dual comb operation. Nanophotonics 2018, 7, 237-242.

(19) Derntl, C. G.; Scalari, G.; Bachmann, D.; Beck, M.; Faist, J.; Unterrainer, K.; Darmo, J. Gain dynamics in a heterogeneous terahertz quantum cascade laser. Appl. Phys. Lett. 2018, 113, 181102.

(20) Burghoff, D.; et al. Terahertz laser frequency combs. Nat. Photonics 2014, 8, 462-467.

(21) Yang, Y.; Burghoff, D.; Reno, J.; Hu, Q. Achieving comb formation over the entire lasing range of quantum cascade lasers. Opt. Lett. 2017, 42, 3888-3891.

(22) Wang, F.; et al. Short Terahertz pulse generation from a dispersion compensated modelocked semiconductor laser. Laser Photonics Rev. 2017, 11, 1700013.

(23) Mezzapesa, F. P.; et al. Tunable on-chip dispersion compensation of broadband THz QCL frequency combs. Opt. Express 2019, 27, 20231-20240.

(24) Consolino, L.; et al. Fully phase-stabilized quantum cascade laser frequency comb. Nat. Commun. 2019, 10, 2938.

(25) Brida, D.; et al. Ultrafast collinear scattering and carrier multiplication in graphene. Nat. Commun. 2013, 4, 1987.

(26) Sun, Z.; et al. Graphene mode-locked ultrafast laser. ACS Nano 2010, 4, 803-810.

(27) Bonaccorso, F.; et al. Production and processing of graphene and $2 \mathrm{~d}$ crystals. Mater. Today 2012, 15, 564-589. 
(28) Ferrari, A. C.; et al. Science and technology roadmap for graphene, related two-dimensional crystals, and hybrid systems. Nanoscale 2015, 7, 4598-4810.

(29) Tredicucci, A.; Vitiello, M. S. Device concepts for graphenebased Terahertz photonics. IEEE J. Sel. Top. Quantum Electron. 2014, 20, 8500109.

(30) Bianchi, V.; et al. Terahertz saturable absorbers from liquid phase exfoliation of graphite. Nat. Commun. 2017, 8, 15763.

(31) Zaugg, C. A.; et al. Ultrafast and widely tuneable verticalexternal-cavity surface-emitting laser, mode-locked by a grapheneintegrated distributed Bragg reflector. Opt. Express 2013, 21, 3154831559 .

(32) Li, H.; et al. Graphene coupled Terahertz semiconductor lasers for enhanced passive comb operation. Advanced Science 2019, 6, 1900460.

(33) Bachmann, D.; et al. Short pulse generation and mode control of broadband terahertz quantum cascade lasers. Optica 2016, 3, 1087-1094.

(34) Wang, F.; et al. Ultrafast response of harmonic modelocked THz lasers. Light: Sci. Appl. 2020, 9, 51.

(35) Gellie, P.; et al. Injection-locking of terahertz quantum cascade lasers up to $35 \mathrm{GHz}$ using RF amplitude modulation. Opt. Express 2010, 18 (20), 20799-20816.

(36) Khurgin, J. B.; Dikmelik, Y.; Hugi, A.; Faist, J. Coherent frequency combs produced by self frequency modulation in quantum cascade lasers. Appl. Phys. Lett. 2014, 104, 081118.

(37) Burghoff, D.; Yang, Y.; Hayton, D. J.; Gao, J.-R.; Reno, J. L.; $\mathrm{Hu}, \mathrm{Q}$. Evaluating the coherence and time-domain profile of quantum cascade laser frequency combs. Opt. Express 2015, 23, 1190.

(38) Riesch, M.; Jirauschek, C. mbsolve: An open-source solver tool for the Maxwell-Bloch equations, https://arxiv.org/abs/2005.05412.

(39) Razavi, B. A study of injection locking and pulling in oscillators. IEEE J. Solid-State Circuits 2004, 39, 1415-1424.

(40) Baryshev, A.; et al. Phase locking and spectral linewidth of a two-mode terahertz quantum cascade laser. Appl. Phys. Lett. 2006, 89, 31115 .

(41) Consolino, L.; et al. Quantum cascade laser based hybrid dual comb spectrometer. Communications Physics 2020, 3, 1-9.

(42) Sterczewski, L. A.; et al. Terahertz hyperspectral imaging with dual chip-scale combs. Optica 2019, 6, 766. 